\title{
Application of hyperosmotic agent to determine gastric cancer with optical coherence tomography ex vivo in mice
}

\author{
Honglian Xiong \\ Zhouyi Guo \\ Changchun Zeng \\ South China Normal University \\ Key Laboratory of Laser Life Science of Ministry \\ of Education \\ Lab of Photonic Chinese Medicine \\ Guangzhou, Guangdong 510631 \\ China
}

\author{
Like Wang \\ Harbin Institute of Technology \\ Graduate School at Shenzhen \\ Shenzhen 518055 \\ China
}

\author{
Yonghong He \\ Tsinghua University \\ Graduate School at Shenzhen \\ Shenzhen 518055 \\ China
}

\author{
Songhao Liu \\ South China Normal University \\ School for Information and Optoelectronic Science \\ and Engineering \\ Guangzhou 510631 \\ China
}

\begin{abstract}
Noninvasive tumor imaging could lead to the early detection and timely treatment of cancer. Optical coherence tomography (OCT) has been reported as an ideal diagnostic tool for distinguishing tumor tissues from normal tissues based on structural imaging. In this study, the capability of OCT for functional imaging of normal and tumor tissues based on time- and depth-resolved quantification of the permeability of biomolecules through these tissues is investigated. The orthotopic graft model of gastric cancer in nude mice is used, normal and tumor tissues from the gastric wall are imaged, and a diffusion of $20 \%$ aqueous solution of glucose in normal stomach tissues and gastric tumor tissues is monitored and quantified as a function of time and tissue depth by an OCT system. Our results show that the permeability coefficient is $(0.94 \pm 0.04) \times 10^{-5} \mathrm{~cm} / \mathrm{s}$ in stomach tissues and $(5.32 \pm 0.17) \times 10^{-5} \mathrm{~cm} / \mathrm{s}$ in tumor tissues, respectively, and that tumor tissues have a higher permeability coefficient compared to normal tissues in optical coherence tomographic images. From the results, it is found that the accurate and sensitive assessment of the permeability coefficients of normal and tumor tissues offers an effective OCT image method for detection of tumor tissues and clinical diagnosis. (-) 2009 Society of Photo-Optical Instrumentation Engineers. [DOI: 10.1117/1.3103341]
\end{abstract}

Keywords: hyperosmotic agent; permeability coefficient; tumor tissues; MCG-803 cells; glucose; optical coherence tomography.

Paper 08401R received Nov. 13, 2008; revised manuscript received Jan. 19, 2009 ; accepted for publication Feb. 8, 2009; published online Mar. 24, 2009.

\section{Introduction}

Optical coherence tomography (OCT) is used in detection and location of varied tumor tissues. Currently, OCT images of tumor tissues have been reported in vitro and in vivo. ${ }^{1-5}$ OCT, as a noninvasive imaging technique, is based on detecting backscattering from intrinsic biological tissues. There is a deformed construction and morphology in tumor tissues, and many experiments showed that signal attenuation in tumor tissues was more evident compared with normal tissues, ${ }^{6}$ because the intrinsic optical properties of biological tissues, such as light absorption and light scattering, are the main factors that limit image contrast of normal and pathological tissues. ${ }^{7}$ However, there are some questions with the OCT imaging of tumor tissues, when there are less differences in light scattering between the normal and tumor tissues, such as earlier cancer, poorly differentiated tissues, and so on, with the small alteration in microstructure and morphology.

A method to change light transport deep in target areas of tissue would improve both therapeutic and diagnostic laser

Address all correspondence to: Changchun Zeng, Lab of Photonic Chinese Medicine, Key Laboratory of Laser Life Science of Ministry of Education, South China Normal University, Guangzhou 510631, China. Tel: +86-20-852138978718; Fax: +86-20-85213017; E-mail: gzzysys@scnu.edu.cn applications. A hyperosmotic agent was utilized in many studies to increase the depth of light penetration into highly scattering tissues for the imaging technique of near-infrared optical coherence tomography. ${ }^{8,9}$ Research has shown that glycerol as a hyperosmotic chemical agent can reduce the amount of refractive mismatch found in the tissue and markedly reduces random scattering, thereby making the skin less turbid for visible wavelengths for a controlled period of time. ${ }^{10}$ The application of glycerol reduced light scattering and increased depth of visibility with optical coherence tomography, and the intensity images indicated changes in blood optical properties and improved contrast of skin crosssection glycerol application. ${ }^{11}$ The mixed hyperosmotic solution of $50 \%$ glycerol and 20\% DMSO has been studied and it was found that the clearing effect of glycerol is enhanced by adding DMSO into it for its synergistic effect. ${ }^{12}$ Many studies showed that hyperosmotic agents could have potential applications for in-vivo low-level light imaging techniques. ${ }^{13,14}$

Dynamic diffusion of the hyperosmotic agent in tissues was observed, and it is found that there is a two-stage diffusion of the hyperosmotic agent: the first stage of diffusion is from the top tissue to the intercellular space, and the second is

$1083-3668 / 2009 / 14(2) / 024029 / 5 / \$ 25.00$ ○ 2009 SPIE 
into the cell matrix. During the first stage, the imaging contrast can be improved by dehydration. ${ }^{13}$ The capability of OCT for functional imaging of normal and atherosclerotic aortic tissues was explored based on time- and depth-resolved quantification of the permeability of biomolecules through these tissues. It is found that the assessment of the permeability coefficients of various physiologically neutral glucose biomolecules in vascular tissues could assist in early diagnosing and detecting the different components of atherosclerotic lesions. And the capability of OCT for noninvasive and nondestructive monitoring and quantifying of permeability coefficients of different analytes and drugs in various biological tissues was studied. ${ }^{15,16}$ To explore the property of permeability coefficients of hyperosmotic agents in tumor tissues and improve the application of OCT in the diction of tumor tissues, we monitor and quantify the diffusion of $20 \%$ aqueous solution of glucose in tumor tissues. The results offer an effective method for clinical diagnosis and detection of tumor tissues.

\section{Materials and Methods}

\subsection{System of Optical Coherence Tomography}

The OCT system used in this study mainly includes resource, fiber conduction, reference arm, and sample arm modules. Briefly, a broadband light source emitted from a superluminescent diode is the central wavelength at $830 \mathrm{~nm}$ with a bandwidth of $50 \mathrm{~nm}$. The light is delivered via a single-mode fiber with a mode field diameter of $5.3 \mu \mathrm{m}$. This OCT system provides an axial resolution of 10 to $15 \mu \mathrm{m}$. The transverse resolution of the system is about $20 \mu \mathrm{m}$, determined by the focal spot size produced by the probe beam. The signal-tonoise ratio (SNR) of the system is measured at $100 \mathrm{db}$. A visible light source $(\lambda=645 \mathrm{~nm})$ is used to guide the probe beam. The OCT system operation is controlled automatically by computer. The detector current is demodulated using a lock-in amplifier and a low-pass filter in software prior to storage. Each in-depth scanning (A-scan) consists of 10,000 data points. Lateral scanning (B-scan) image is obtained by moving the mirror relative to the tissue sample, which takes about $1.0 \mathrm{~s}$. The data acquisition software is written in LabView 7.2-D. OCT images are obtained in each experiment and stored in a PC for further processing.

\subsection{Material and Animal Tumor Model}

$20 \mathrm{BALB} / \mathrm{c}$ nude mice weighing between $15 \mathrm{~g}$ and $18 \mathrm{~g}$, purchased from the Laboratory Animal Center at Sun Yat-Sen University, were used in this study. Animals were maintained under specific pathogen-free conditions, in which chow and tap water were available ad libitum. The animals were divided into two groups: the normal control group (ten samples) and the tumor model group (ten samples). The tumor model group received an orthotopic (gastric mucosa) graft operation.

The human gastric cancer cell line MCG-803 cells (CTCC) were cultured in DMEM culture medium supplemented with $10 \%$ fetal calf serum, $50-\mathrm{U} / \mathrm{ml}$ penicillin, and $50-\mu \mathrm{g} / \mathrm{ml}$ streptomycin. Suspensions of MCG-803 cells (5 to $10 \times 10^{6}$ viable cells/mouse) were implanted into the right dorsal portion of BALB/c nude mice. After 20 days, the inoculated tu- mors reached diameters of about 3 to $6 \mathrm{~mm}$, the mice were killed, and tumor tissues were surgically extracted for establishing the animal model of gastric carcinoma in situ. The resected tumor tissues were sheared to small tumor tissues about one cubic millimeter. The ten mice in the tumor model group were opened at their abdominal cavity by operation, the gastric walls were exposed, and then the small tumor tissues were implanted into the gastric wall. After 20 days, when the diameter of the implanted tumors became about 2 to $4 \mathrm{~mm}$, the animals were prepared for OCT imaging.

Then, the gastric walls in the normal control group and the tumor model group were cut into approximately $1 \times 1-\mathrm{cm}$ samples and placed in 4-ml $0.9 \%$ saline solution. Continuous OCT imaging was performed for approximately $1 \mathrm{~h}$ at $22^{\circ} \mathrm{C}$. The region was imaged for approximately 7 to $10 \mathrm{~min}$ before the glucose solution was applied to establish a baseline for the OCT signal slope graph. Then 4-ml 40\% glucose solution was added to the saline solution in a 1:1 ratio to maintain the end level of $20 \%$ glucose.

\subsection{Methods of Measurement and Calculation}

The permeability coefficients of glucose in tissues ex vivo were measured by monitoring slope changes of OCT signals in the tissue. The permeability coefficient of glucose in the tissues was computed with the OCT signal slope (OCTSS). ${ }^{17}$ The glucose that diffused through the tissues changed the local optical property, which was detected by the OCT system. Glucose penetrated through the tissue to replace water in tissues. Due to the increase of in-depth concentration, the light scattering coefficient is altered. The changes of the optical properties were shown in biological tissues through time induced by the glucose diffusion.

The normal stomach mucosa tissues and stomach tissues are performed by OCT at a central length of $830 \mathrm{~nm}$. 2-D OCT images were acquired by continuous imaging of the tissue. 20 consecutive scans were averaged to obtain OCT intensive profiles as a function of depth. A region with minimal alterations in the OCT signal was selected for time-dependent analysis of its optical properties as glucose diffused through these tissues. The permeability coefficient of glucose in the normal stomach and tumor tissues was calculated by using the following equation:

$$
P=\frac{z}{t}
$$

where $P$ is the permeability coefficient, $z$ is the thickness of the chosen section, and $t$ is the time for the glucose to diffuse through that section. ${ }^{17}$

\subsection{Statistical Analysis}

Data are presented as a mean \pm SD for a number of sample animals. Data were analyzed by Student's t-tests for unpaired data. $\mathrm{P}<0.05$ was considered statistically significant. All statistical analyses were performed with the statistics software SPSS 10.0 for Windows. 


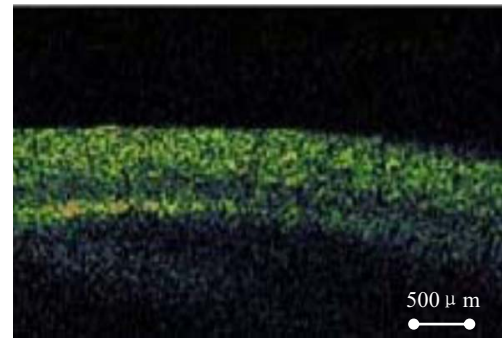

(a)

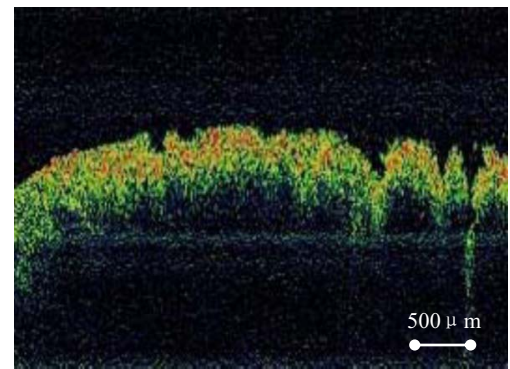

(d)

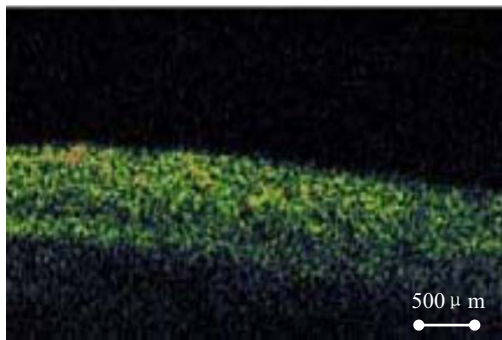

(b)

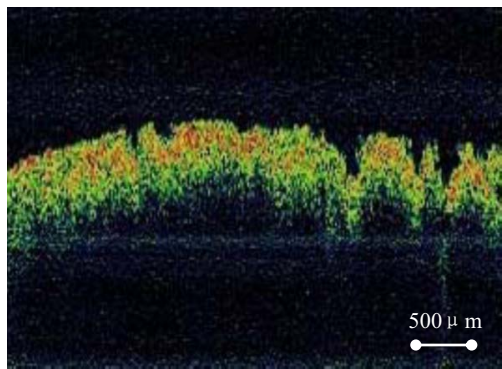

(e)

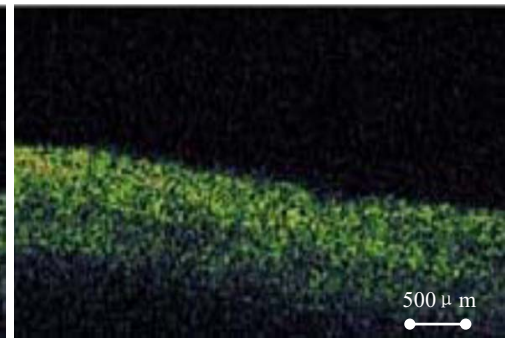

(c)

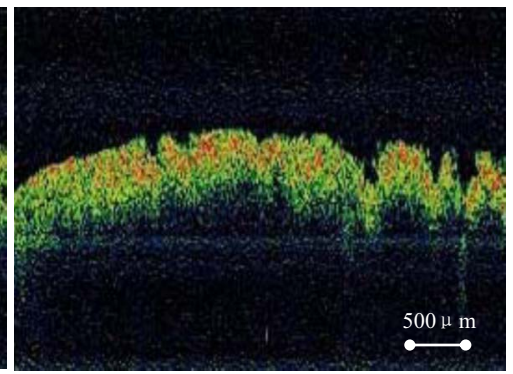

(f)

Fig. 1 OCT images of (a), (b), and (c) the normal stomach, and (d), (e), and (f) tumor tissues at 0, 20, and 40 min during the $20 \%$ glucose diffusion experiment.

\section{Results}

3.1 Optical Coherence Tomography Images of Normal Stomach Tissues and Stomach Tumor Tissues

Figures 1(a)-1(c) show the OCT images of the normal stomach tissues ex vivo at 0,20 , and 40 min during the $20 \%$ glucose diffusion experiment. It is shown that the submucosal layers of normal stomach tissues are visible with layer structures at 0 and $20 \mathrm{~min}$. The imaging depth is improved after the application of $20 \%$ glucose. Figures $1(\mathrm{~d})-1(\mathrm{f})$ display the OCT image of the stomach tumor tissues ex vivo. It is shown that there are no layer structures in the gastric cancer tissues in the tumor model group. It can be seen that there is an improvement in the imaging depth after the application of $20 \%$ glucose.

\subsection{Optical Coherence Tomography Signal Slope Graphs of Normal Stomach Tissues and Stomach Tumor Tissues}

Figure 2(a) shows a typical OCTSS graph in the normal stomach tissues during a glucose diffusion experiment. The sample was monitored for approximately $10 \mathrm{~min}$ before glucose solution was applied. The monitored region was about $135 \mu \mathrm{m}$ thick and $195 \mu \mathrm{m}$ away from the surface. From Fig. 2(a),

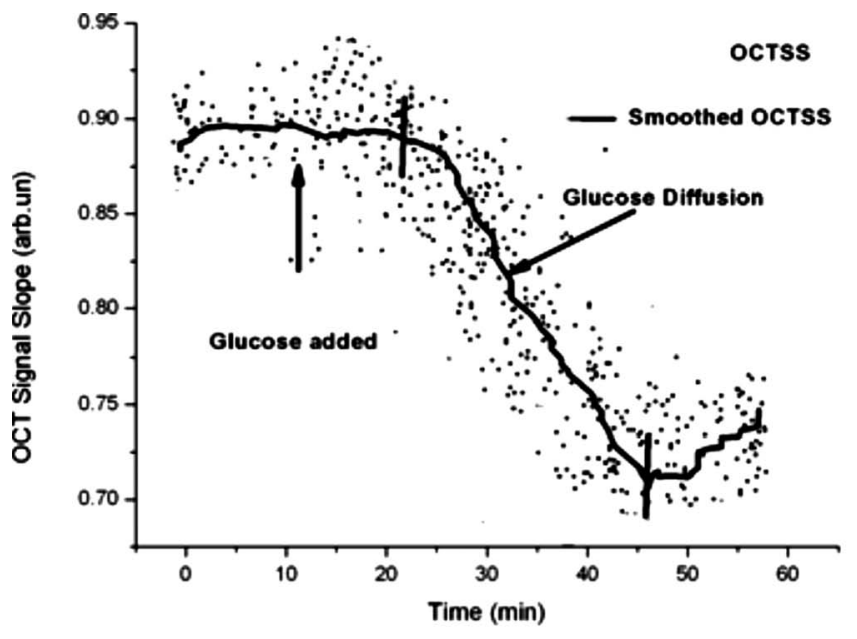

(a)

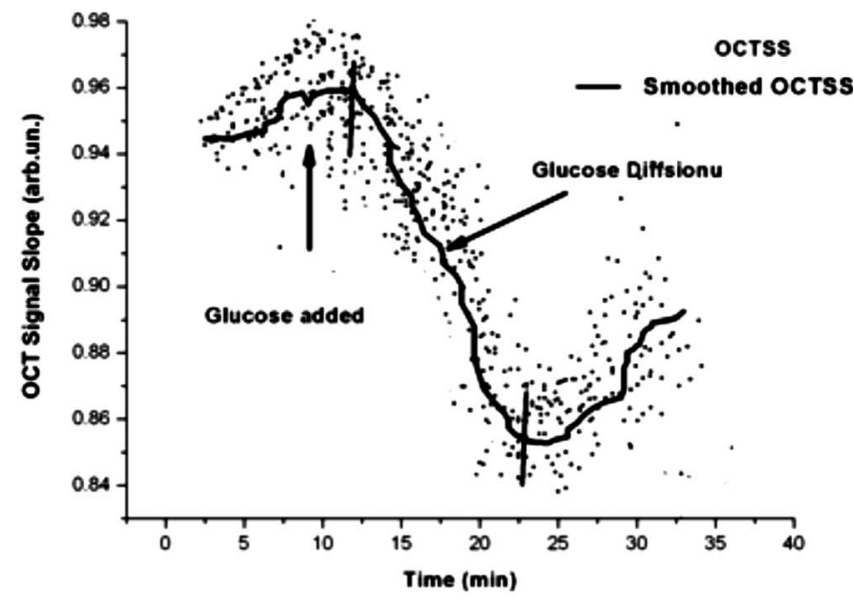

(b)

Fig. 2 OCTSS graphs as a function of time from (a) normal stomach tissues and (b) stomach tumor tissues during the glucose $20 \%$ diffusion experiment. The tick marks indicate the interval of glucose diffusion. 


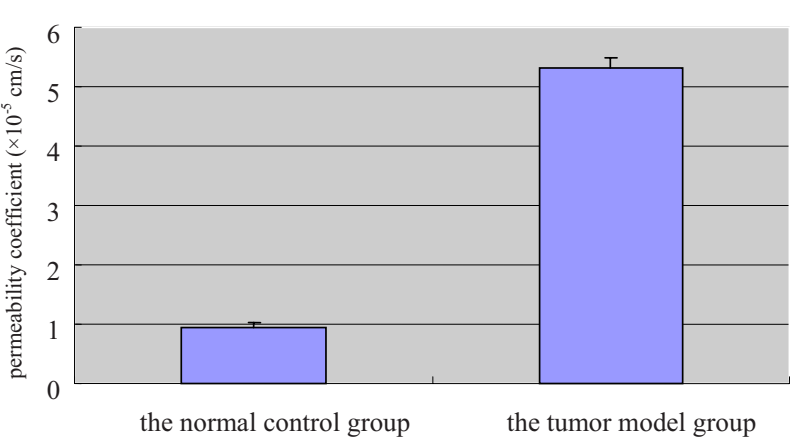

Fig. 3 Comparison of permeability coefficient of glucose diffusion.

there was a decrease of the OCT signal slope caused by a reduction of scattering in the tissues due to the local increase of glucose concentration. $20 \%$ glucose solution reached the monitored region approximately 20 min after administration. This decrease of the slope could be due to the concentration gradient differences between both sides of the tissue; the fluid (mainly water) moved from areas of high concentration to those of lower concentration. And the monitored region was completely diffused through by the $20 \%$ glucose solution. At this time, a reverse process, the increase of OCT signals, in the OCTSS was seen. Water diffused back into the tissues due to the difference, which means water was diffusing back into the tissue due to the difference of the concentration gradient of the glucose solution on both sides. After $20 \mathrm{~min}$, equilibrium is reached. There is no evident change in the graph. The permeability coefficient of $20 \%$ glucose from different experiments was found to be $(0.94 \pm 0.04) \times 10^{-5} \mathrm{~cm} / \mathrm{s}$. Figure 2(b) displays an OCTSS graph during a $20 \%$ glucose diffusion experiment in the tumor tissues. The same procedure was used in the tumor tissue experiments. The tumor tissue region monitored was $310 \mu \mathrm{m}$ thick and $420 \mu \mathrm{m}$ away from the surface. The permeability coefficient of $20 \%$ glucose in the tumor tissues was found to be $(5.32 \pm 0.17) \times 10^{-5} \mathrm{~cm} / \mathrm{s}$.

Comparing the permeability coefficient in the tumor model group with that in the normal control group, it is found that there is a significant difference in the permeability coefficient of glucose between tumor tissues and normal tissues ( $p$ $<0.01$ ) (see Fig. 3).

\section{Discussion}

OCT techniques were used for detecting the changes in structure associated with early neoplastic diseases. OCT-based endoscopic systems perform imaging of specific target areas, such as the breast duct, the urinary tract, and so on. The gastrointestinal tract is the first internal organ for which endoscopic OCT was applied for study. ${ }^{18}$ In clinical trials, OCT endoscopes have been used in malignancies in different organs, including the gastrointestinal tract, larynx, esophagus, uterine cervix, colon, urinary bladder, and vascular, systems. ${ }^{18-20}$ In this researches, it is shown that the alteration of intrinsic light scattering properties in tumor tissues make detection possible by OCT imaging, and can promote the clinical application of OCT technique.

In this study, gastric cancer models in mice were established, normal stomach tissues and gastric tumor tissues were detected by OCT imaging, and a diffusion of $20 \%$ aqueous solution of glucose in normal stomach tissues and gastric tumor tissues was monitored and quantified. By gathering interference data at points across the surface, at cross sectional tissues, and averaging the OCT profile images, the optical properties of tissues are measured. To measure the permeability coefficient, the refractive index is assumed to be equal to 1.40 in gastric wall tissues. The diffusion of glucose will change the refractive index during the course of the experiment, and therefore will affect the size of the region selected for calculations. But, this alteration in tissue optical size was small enough and was neglected in our computation. Also, biological tissue is a turbid nonuniform tissue with some variations in the refractive indexes through its layers. Our results show that the permeability coefficient of stomach tumor tissues is higher than that of normal stomach tissues significantly. These indicate that OCT has the capability to measure the permeability coefficient of biological tissues and differentiate tumor tissues from normal constructive tissues. The passive diffusion of macromolecules in tissues is critically dependent on the amount and structural organization that could vary spatially within a specific tissues segment and from normal to tumor tissues according to the disease stage. ${ }^{21}$ The alteration of permeability coefficient of hyperosmotic agent diffusion in tumor tissues might provide a basis for tissue characterization and early tumor diagnosis. Therefore, monitoring and quantifying the diffusion of a hyperosmotic agent by OCT imaging could assist in the diagnosis and early detection of tumors, and glucose solution, one important molecule naturally occuring in blood and having no side effects, could be easily applied in future clinical applications.

\section{Conclusion}

OCT's capability of nondestructive quantification of glucose diffusion in biological tissues makes it an excellent imaging tool in studying drug and analyte diffusion. It is shown here that the permeability coefficient in abnormal tissues is found to be far bigger than that in normal ones. From the permeability coefficient of normal stomach tissues and tumor tissues, it is found that there is a clear difference in composition and structure. The results presented confirm the ability of OCT in biological tissue imaging and diagnosing, as well as detecting tumor tissues. The methods enable accurate assessment of analyte diffusion and assist in the early diagnosis of various tumor tissues.

\section{Acknowledgments}

The authors would like to acknowledge financial support from the National Natural Science Foundation of China (grant numbers 30600797 and 30873238), and the Key Science and Technology Project of Guangdong Province of China (grant numbers 2006B35602001, 2007B031401010, and 2008B090500125).

\section{References}

1. A. Boppart, A. Goodman, J. Libus, C. Pitris, C. A. Jesser, M. E. Brezinski, and J. G. Fujimoto, "High resolution imaging of endometriosis and ovarian carcinoma with optical coherence tomography: feasibility for laparoscopic-based imaging," Br. J. Obstet. Gynaecol. 106(10), 1071-1077 (1999).

2. J. M. Olmedo, K. E. Warschaw, J. M. Schmitt, and D. L. Swanson, "Correlation of thickness of basal cell carcinoma by optical coher- 
ence tomography in vivo and routine histologic findings: a pilot study," Dermatol. Surg. 33(4), 421-426 (2007).

3. M. T. Tsai, H. C. Lee, C. K. Lee, C. H. Yu, H. M. Chen, C. P. Chiang, C. C. Chang, Y. M. Wang, and C. C. Yang "Effective indicators for diagnosis of oral cancer using optical coherence tomography," Opt. Express 16(20), 15847-15862 (2008).

4. G. J. Tearney, M. E. Brezinski, B. E. Bouma, S. A. Boppart, C. Pitris, J. F. Southern, and J. G. Fujimoto, "In vivo endoscopic optical biopsy with optical coherence tomography," Science 276(5321), 2037-2039 (1997).

5. C. A. Jesser, S. A. Boppart, C. Pitris, D. L. Stamper, G. P. Nielsen, M. E. Brezinski, and J. G. Fujimoto, "High resolution imaging of transitional cell carcinoma with optical coherence tomography: feasibility for the evaluation of bladder pathology," Br. J. Radiol. 72(864), 1170-1176 (1999).

6. C. Pitris, M. E. Brezinski, B. E. Bouma, G. J. Tearney, J. F. Southern, and J. G. Fujimoto, "High resolution imaging of the upper respiratory tract with optical coherence tomography-Đ a feasibility study," Am. J. Respir. Crit. Care Med. 157, 1640-1644 (1998).

7. D. Huang, E. A. Swanson, C. P. Lin, J. S. Schuman, W. G. Stinson, W. Chang, M. R. Hee, T. Flotte, K. Gregory, C. A. Puliafito, and J. G. Fujimoto, "Optical coherence tomography," Science 254, 1178-1181 (1991).

8. G. Vargas, E. K. Chan, J. K. Barton, H. G. Rylander, and A. J. Welch, "Use of an agent to reduce scattering in skin," Lasers Surg. Med. 24(2), 133-141 (1999).

9. G. Vargas, A. Readinger, S. S. Dozier, and A. J. Welch, "Morphological changes in blood vessels produced by hyperosmotic agents and measured by optical coherence tomography," Photochem. Photobiol. 77(5), 541-549 (2003).

10. O. F. Stumpp, A. J. Welch, T. E. Milner, and J. Neev, "Enhancement of transepidermal skin clearing agent delivery using a $980 \mathrm{~nm}$ diode laser," Lasers Surg. Med. 37(4), 278-285 (2005).

11. V. V. Tuchin, X. Xu, and R. K. Wang, "Dynamic optical coherence tomography in studies of optical clearing, sedimentation, and aggregation of immersed blood," Appl. Opt. 41(1), 258-271 (2002).

12. X. Xu and R. K. Wang, "Synergistic effect of hyperosmotic agents of dimethyl sulfoxide and glycerol on optical clearing of gastric tissue studied with near infrared spectroscopy," Phys. Med. Biol. 49(3), 457-468 (2004).

13. Y. He and R. K. Wang, "Dynamic optical clearing effect of tissue impregnated with hyperosmotic agents and studied with optical coherence tomography," J. Biomed. Opt. 9(1), 200-206 (2004).

14. Y. He and R. K. Wang, "Improvement of low-level light imaging performance using optical clearing method," Biosens. Bioelectron. 20(3), 460-467 (2004)

15. M. G. Ghosn, V. V. Tuchin, and K. V. Larin, "Depth-resolved monitoring of glucose diffusion in tissues by using optical coherence tomography," Opt. Lett. 31(15), 2314-2316 (2006).

16. M. G. Ghosn, E. F. Carbajal, N. A. Befrui, A. Tellez, J. F. Granada and K. V. Larin, "Permeability of hyperosmotic agent in normal and atherosclerotic vascular tissues," J. Biomed. Opt. 13(1), 010505 (2008).

17. M. G. Ghosn, V. V. Tuchin, and K. V. Larin, "Nondestructive quantification of analyte diffusion in cornea and sclera using optical coherence tomography," Invest. Ophthalmol. Visual Sci. 48(6), 27262733 (2007).

18. Y. Chen, A. D. Aguirre, P. L. Hsiung, S. Desai, P. R. Herz, M. Pedrosa, Q. Huang, M. Figueiredo, S. W. Huang, A. Koski, J. M. Schmitt, J. G. Fujimoto, and H. Mashimo, "Ultrahigh resolution optical coherence tomography of Barrett's esophagus: preliminary descriptive clinical study correlating images with histology," Endoscopy 39(7), 599-605 (2007).

19. E. S. Matheny, N. M. Hanna, W. G. Jung, Z. Chen, P. Wilder-Smith, R. Mina-Araghi, and M. Brenner, "Optical coherence tomography of malignancy in hamster cheek pouches," J. Biomed. Opt. 9(5), 978981 (2004)

20. G. Zuccaro, N. Gladkova, J. Vargo, F. Feldchtein, E. Zagaynova, D. Conwell, G. Falk, J. Goldblum, J. Dumot, J. Ponsky, G. Gelikonov, B. Davros, E. Donchenko, and J. Richter, "Optical coherence tomography of the esophagus and proximal stomach in health and disease," Am. J. Gastroenterol. 96(9), 2633-2639 (2001).

21. C. P. Winlove, K. H. Parker, N. C. Avery, and A. J. Bailey, "Interactions of elastin and aorta with sugars in vitro and their effects on biochemical and physical properties," Diabetologia 39(10), 11311139 (1996). 